Proyecciones Journal of Mathematics

Vol. 29, No 1, pp. 49-56, May 2010.

Universidad Católica del Norte

Antofagasta - Chile

\title{
A NOTE ON THE UPPER RADICALS OF SEMINEARRINGS
}

\author{
MUHAMMAD ZULFIQAR \\ GOVT. COLLEGE UNIVERSITY LAHORE, PAKISTAN \\ Received: June 2009. Accepted : December 2009
}

\begin{abstract}
In this paper we work in the class of seminearrings. Hereditary properties inherited by the lower radical generated by a class $M$ have been considered in [2, 5, 6, 7, 9, 10, 12]. Here we consider the dual problem, namely strong properties which are inherited by the upper radical generated by a class $M$.
\end{abstract}




\section{Introduction and Preliminaries}

V. G. Van Hoorn and B. Van Rootselaar [11] discussed general theory of seminearrings. The theory was further enriched by many authors (see [1, $3,4,13,14])$. The upper radicals were investigated by (see $[2,9,12])$ for radical classes of rings. Here we are interested in generalizing several results from $[2,5,6,7,9,10,12]$ in the framework of seminearring, which is quite different from the ring theoretical approach discussed in $[2,5,6,7$, $9,10,12]$. Throughout this paper $N$ will denote an seminearrings and $\omega$ be the universal class of all seminearrings. An semi-ideal $I$ of $N$ is denoted by $I \leq N$. In the following we shall be working within the class of all seminearrings.

Consider non-associative seminearrings as general algebras $(N,+,$.$) ,$ where $(N,+)$ is a semigroup, $(N,$.$) is a groupoid, and only the one-side$ distributive law holds.

Lower radical classes for seminearrings can be constructed similar to the construction of lower radicals for rings (see $[2,5,6,7,8,9,10,12,15,16]$ ). First we include necessary preliminary, let $\omega$ be the universal class of all seminearrings and $M$ be a sub-class of $\omega$ and let $M_{0}$ be the homomorphic closure of $M$ in $\omega$. For each $N \varepsilon \omega$, let $D_{1}(N)$ be the set of all semi-ideals of $N$. Inductively we define

$D_{n+1}(N)=\left\{I: I\right.$ is an semi-ideal of some seminearring in $\left.D_{n}(N)\right\}$

Let $D(N)=\bigcup_{n \in N} D_{n}(N), \mathrm{n}=1,2,3$. . . By using rings theoretical approach discussed in [12], we have

$$
£ M=\left\{N \varepsilon \omega: D(N / I) \cap M_{0} \neq 0, \text { for each proper semi }- \text { ideal I of } N\right\},
$$

is the Lee construction for lower radical determined by $M$, and $M \subseteq £ M$ (see also $[8,15,16])$.

First we give a construction for the upper radical, dual to the construction of [10] for the lower radical. From this the theorem on the inheritance of the left strong property is deduced.

We define the following classes from a given class $M$ of seminearrings: $I M=\{N: N$ is a subsemi-ideal of some seminearring of $M\}$; 
$T M=\{N: N$ contains an semi-ideal $B$ such that $B \varepsilon M$ and $N / B \varepsilon M\} ;$

$S M=\left\{N: N\right.$ contains a descending chain of semi-ideals $B_{i}$ such that $B_{i}=0$ and $\left.N / B_{i} \varepsilon M\right\}$.

It is clear that $M$ is contained in $I M$ and $S M$ and that $M$ is contained in $T M$ if 0 belongs to $M$. The class $M$ is semi-ideally closed if and only if $M$ $=I M$. If $M$ is semi-ideally closed then it follows easily that $T M$ and $S M$ are also semi-ideally closed. For undefined terms of seminearrings we may refer (see $[1,3,4,9,11,12,13,14,16])$.

\section{Upper Radicals}

We extend the results of $[2,5,6,7,9,10,12]$ by using the above construction of upper radical for seminearring which is indeed provides an excellent and different approach to handle the many results of $[2,5,6,7$, $9,10,12]$ in the frame work of seminearring.

Definition 2.1. If $\rho$ is a radical class of seminearring then it admits a semisimple class:

$$
S \rho=\{N \varepsilon \omega: \rho(N)=0\} .
$$

The following theorems were proved by N. J. Divinsky [2] for rings. Here we generalize it for seminearring which can be obtained on the line of rings theoretical approach discussed in [2].

Theorem 2.2. For any radical property $N$, every semi-ideal of an $N$ semisimple is itself $N$-semisimple.

Proof. The proof of our Theorem 2.2 is very similar to the proof of [2].

Theorem 2.3. The class $M$ is the class of all $S$-semisimple seminearrings with respect to some radical property $S$ if and only if $M$ satisfies the following conditions:

(1) Every non-zero semi-ideal of a seminearring of $M$ can be mapped homomorphically on to some non-zero seminearring of $M$.

(2) If every non-zero semi-ideal of a seminearring $N$ can be mapped homomorphically onto some non-zero seminearring of $M$, then the seminearring 
$N$ must be in $M$.

Proof. The proof of our Theorem 2.3 is very similar to the proof of [2].

Theorem 2.4. A non-empty class of seminearrings $M$ is the semisimple class with respect to some radical if and only if $M=I M=T M=S M$.

Proof. By Theorem 2.2 a semisimple class is semi-ideally closed. Also if $B$ and $N / B$ are semisimple and $\rho(N)$ is the radical of $\mathrm{N},(\rho(N)+B) / B$ is semisimple, being an semi-ideal of $N / B$ and is also radical being isomorphic to $\rho(N) /\left(\rho(N) \cap B^{*}\right.$ ), (where $B^{*}$ is a k-semi-ideal generated by $B$ (see [8, $15,16])$. Hence $\rho(N) \subseteq B$. As $B$ is semisimple we have $\rho(N)=0$. Therefore $N$ is semisimple. If $B_{i}$ is a family of semi-ideals of $N$ such that $N / B_{i}$ is semisimple and $\cap B_{i}=0$, then as above, $\rho(N) \subseteq B_{i}$ and so $\rho(N) \subseteq \cap B_{i}$. Therefore $N$ is semisimple. It follows that a semisimple class $M$ satisfies $M=I M=T M=S M$.

Conversely let $M$ be a class of seminearrings such that $M=I M=$ $T M=S M$. We show that $M$ is a semisimple class by verifying the conditions (1) and (2) of Theorem 2.3. Since $M=I M$ condition (1) of Theorem 2.3 is clear. Now let $N$ be a seminearring such that every nonzero semi-ideal of $N$ can be mapped onto some non-zero seminearring of $M$. To complete the proof we must show that $N$ is in $M$. Consider the family of proper semi-ideals $G$ of $N$ such that $N / G \varepsilon M$. From $M=S M$ and using Zorn's Lemma we see that there is an semi-ideal $B$ minimal in this family. If $B=0$, we are finished. If not there is an semi-ideal $B$ minimal in the family of proper semi-ideals of $B$ whose quotients belong to $M . J$ is not an semi-ideal of $N$. Since $M=T M$ would then imply $N / J \varepsilon M$, contradicting the minimality of $B$. Hence either $N J J$ or $J N J$. We may assume without loss of generality that there exists $n \varepsilon N$ with $n J J$. Consider $(n J+J) / J$. We have $n J B \subseteq n J$ and $B n J \subseteq B J \subseteq J$. Hence $(n J+J) / J$ in an semi-ideal of $B / J$. Since $B / J \varepsilon M$ and $I M=M$ we have $(n J+J) / J \varepsilon M$. Consider the mapping from $J$ to $(n J+J) / J$ given by $\eta(x)=n x+J, \eta(x y)=n x y+J \subseteq N J J+J \subseteq B J+J \subseteq J$; $\eta(x) \eta(y)=n x n y+J \subseteq N J N J+J \subseteq B J+J \subseteq J$. Therefore $\eta$ is an epimorphism. Let $K$ be the kernel of $\eta$, i.e. $K=\{x \varepsilon J: n x \varepsilon J\}$. Let $x \varepsilon K$, $b \varepsilon B$; then $n b x \subseteq B J \subseteq J, n x b \subseteq J B \subseteq J$. Hence $K$ is an semi-ideal of $B$. However we have $B / J \varepsilon M$ and

$$
J / K \cong(n J+J) / J \varepsilon M
$$


Since $T M=M$ it follows that $B / K \varepsilon M$. This contradicts the minimality of $J$. Therefore $N \varepsilon M$ and $M$ is a semisimple class.

Now we can give the upper radical construction. Let $M$ be a non-empty class of seminearrings. Let $M_{1}=I M$. We define $M_{\alpha}$ inductively on ordinals $\alpha>1$ as follows. If $\alpha$ is not a limit ordinal $M_{\alpha}=T M_{\alpha-1}$. If $\alpha$ is a limit ordinal $M_{\alpha}=\mathrm{S}\left(\bigcup_{\beta<\alpha} M_{\beta}\right)$. Finally we set $\bar{M}=\cup M_{\alpha}$.

Theorem 2.5. For any non-empty class $M$ of seminearrings, $\bar{M}$ is the smallest semisimple class containing $M$.

Proof. It is clear that if $M$ is contained in semisimple class so also are $I M, T M$, and $S M$. Therefore $\bar{M}$ is contained in every semisimple class containing $M$. It remains to show that $\bar{M}$ is a semisimple class. It is clear that $I M_{\alpha}=M_{\alpha}$ for all $\alpha$ and hence that $I \bar{M}=\bar{M}$. Let $N \varepsilon T \bar{M}$. Then $\mathrm{N}$ contains an semi-ideal $B$ with $B$ and $N / B$ in $\bar{M}$. Therefore there exists an ordinal $\alpha$ with both $B$ and $N / B \varepsilon M_{\alpha}$. Therefore $N \varepsilon T M_{\alpha}=M_{\alpha+1}$. Hence $N \varepsilon \bar{M}$. Therefore $\bar{M}=T \bar{M}$. Let $N \varepsilon S \bar{M}$. Let $B_{i}$ be the descending chain of semi-ideals of $N$ with $\cap B_{i}=0$ and $N / B \varepsilon \bar{M}$. Then $N / B_{i} \varepsilon M_{\alpha_{i}}$, for some $\alpha_{i}$. Since the indices $I$ form a set there exists a limit ordinal $\alpha$ with $\alpha_{i}$ for all i. Then $N / B_{i} \varepsilon \bigcup_{\beta<\alpha} M_{\beta}$ and so $N \varepsilon S\left(\bigcup_{\beta<\alpha} M_{\beta}\right)=M_{\alpha}$. Therefore $A \varepsilon \bar{M}$ and $\bar{M}=S \bar{M}$. It follows from Theorem 2.4 that $\bar{M}$ is a semisimple class of seminearrings.

Definition 2.6. A radical $\rho$ is said to be left strong, if every radical left semi-ideal of a seminearring $N$ is contained in the radical of $N$. Equivalently semisimple seminearrings contain no non-zero radical left semi-ideal of every seminearring in $M$ has a non-zero image in $I M$. Clearly a radical is strong if and only if its semisimple class is left strong.

Theorem 2.7. If a non-empty class $M$ of seminearrings is left strong then the upper radical generated by $M$ is left strong.

Proof. Let $\rho$ denote the upper radical generated by $M$. By Theorem 2.5 the semisimple class of the radical $\rho$ is $\bar{M}$. We need to show that if $K$ is a non-zero left semi-ideal of a seminearring $N$ in $\bar{M}$ then $K$ has a non-zero image in $\bar{M}$. Let $N \varepsilon M_{\alpha}$. The proof is by transfinite induction on $\alpha$. First suppose that $N \varepsilon M_{1}=I M$. Then $N$ is a subsemi-ideal of a seminearring in $M$. Let $N=N_{1} \subseteq N_{2} \subseteq \ldots \subseteq N_{n}$, where $N_{n} \varepsilon M$ and $N_{i}$ is an semi-ideal of $N_{i+1}$. We prove this case by induction on $\mathrm{n}$. If $\mathrm{n}=1$ the required result 
holds. $K+N_{2} K$ is a left semi-ideal of $N_{2}$. By the inductive assumption $K+N_{2} K$ has a non-zero image $\left(K+N_{2} K\right) / J$ in $\bar{M}$. If $K \subseteq J$ then

$$
K /\left(K \cap J^{*}\right) \cong(K+J) / J
$$

(where $J^{*}$ is a k-semi-ideal generated by $J$ (see $\left.[8,15,16]\right)$ ) is a non-zero semi-ideal of $\left(K+N_{2} K\right) / J$ and so is in $\bar{M}$. If $K \subseteq J$ then there exist $b \varepsilon N_{2}$ with $b K J$; then $(J+b K) / J$ is an semi-ideal of $\left(K+N_{2} K\right) / J$ and so is in $\bar{M}$ . As before, the mapping $\eta$ from $K$ to $(J+b K) / J$ given by $\eta(x)=b x+J$ is an epimorphism and so $K$ has a non-zero image in $M$ as required. Now let $N \varepsilon M_{\alpha}$ and assume that the result has been proved for ordinals less then $\alpha$. If $\alpha$ in not a limit ordinal then $N$ contains an semi-ideal $B$ and $N / B$ in $M_{\alpha-1}$. If $K \subseteq B$ the required result holds. Otherwise

$$
K /\left(K \cap B^{*}\right) \cong(K+B) / B
$$

(where $B^{*}$ is a k-semi-ideal generated by $B($ see $[8,15,16])$ ) which is a non-zero left semi-ideal of $N / B$. Again the required result follows. If $\alpha$ is a limit ordinal then $N$ contains a descending chain of semi-ideals $B i$ with $\cap B i=0$ and $N / B_{i} \varepsilon M_{\alpha_{i}}, \alpha_{i}<\alpha$. For some i, $K B_{i}$. Then $\left(K+B_{i}\right) / B_{i}$ is a non-zero left semi-ideal of $N / B_{i}$. Thus in all cases $K$ has a non-zero image in $\bar{M}$. Therefore the upper radical generated by $M$ is left strong.

Acknowledgement. The author thank the referee for his useful comments and suggestions for the improvement of the paper.

\section{References}

[1] Birkenmeier, G. F., "SEMINEARRINGS AND NEARRINGS" induced by the circle operation, preprint, (1989).

[2] Divinsky, N. J., "RINGS AND RADICALS", Toronto, (1965).

[3] Golan, J. S., "THE THEORY OF SEMIRINGS WITH APPLICATIONS IN MATHEMATICS AND THEORETICAL COMPUTER SCIENCE", Pitman Monographs and Surveys in Pure and Applied Maths. 54, New-York, (1986). 
[4] Hebisch, U. and Weinert, H. J., "SEMIRINGS ALGEBRAIC THEORY AND APPLICATIONS IN COMPUTER SCIENCE", Vol. 5 (Singapore 1998).

[5] Hoffman, A. E. and Leavitt, W. G., "Properties inherited by the Lower Radical", Port. Math. 27, pp. 63-66, (1968).

[6] Krempa, N. J. and Sulinski, A., "Strong radical properties of alternative and associative rings", J. Algebra 17, pp. 369-388, (1971).

[7] Leavitt, W. G., "Lower Radical Constructions', Rings, Modules and Radicals, Budapest, pp. 319-323, (1973).

[8] Olson, D. M. and Jenksins, T. L., "Radical Theory for Hemirings", Jour. of Nat. Sciences and Math., Vol. 23, pp. 23-32, (1983).

[9] Szasz, F. A., "RADICALS OF RINGS", Mathematical Institute Hungarian Academy of Sciences, (1981).

[10] Tangeman, R. L. and Kreiling, D., "Lower radicals in non-associative rings", J. Australian Math. Soc. 14, pp. 419-423, (1972).

[11] Hoorn, V. G. and Rootselaar, B., " Fundamental notions in the theory of seminerarings", Compositio Math. 18, pp. 65-78, (1966).

[12] Wiegandt, R., "RADICAL AND SEMISIMPLE CLASSES OF RINGS", Queens University, Ontarto, Canada, (1974).

[13] Weinert, H. J., "Seminearrings, seminearfield and their semigrouptheoretical background.", Semigroup Forum 24, pp. 231-254, (1982).

[14] Weinert, H. J., "Extensions of seminearrings by semigroups of right quotients", Lect. Notes Math. 998, pp. 412-486, (1983).

[15] Yusuf, S. M. and Shabir, M., "Radical classes and semisimple classes for hemiring", Studia Sci. Math. Hungarica 23, pp. 231-235, (1988).

[16] Zulfiqar, M., "The sum of two radical classes of hemirings", Kyungpook Math. J. Vol. 43, pp. 371-374, (2003). 
Permanent address :

Department of Mathematics

Govt.College University Lahore

Pakistan

e-mail : mzulfiqarshafi@hotmail.com 